\title{
La palatalisation vannetaise
}

Vannetais palatalisation

\section{Erwan Le Pipec}

\section{OpenEdition}

\section{Journals}

Édition électronique

URL : https://journals.openedition.org//bl/1211

DOI : $10.4000 / \mathrm{lbl} .1211$

ISSN : 2727-9383

\section{Éditeur}

Université de Bretagne Occidentale - UBO

\section{Édition imprimée}

Date de publication : 1 juin 2015

Pagination : 297-327

ISBN : 979-10-92331-16-5

ISSN : 1270-2412

\section{Référence électronique}

Erwan Le Pipec, « La palatalisation vannetaise », La Bretagne Linguistique [En ligne], 19 | 2015, mis en ligne le 01 mai 2021, consulté le 22 mai 2021. URL : http://journals.openedition.org//bl/1211 ; DOI : https://doi.org/10.4000/lbl.1211

\section{cc) (7)}

La Bretagne Linguistique est mise à disposition selon les termes de la Licence Creative Commons Attribution 4.0 International. 


\title{
Erwan LE PIPEC*
}

\section{La palatalisation vannetaise}

\begin{abstract}
P
Pour les locuteurs bretonnants d'aujourd'hui, l'idée d'une spécificité vannetaise, constituée par la palatalisation des occlusives vélaires $(/ \mathrm{k}-\mathrm{g} /$ prononcées $[\mathrm{t}-\mathrm{d}]$ ] $)$, relève du sens commun. Au point de voir souvent désigner familièrement le breton de l'ancien pays de Vannes par le surnom de " breton tcheu-tcheu ${ }^{1}$. Une seconde idée reçue veut que cette évolution phonétique soit d'origine romane : le vannetais serait ainsi, soit un breton sous influence française, soit un parler dans lequel se manifesterait la résurgence d'un substrat roman. Ce qui est curieux, c'est qu'il n'y a pas si longtemps que cette caractéristique est mise en avant. Les lexicographes du XVIII ${ }^{\mathrm{e}}$ siècle, qui donnent des indications précises sur les différences entre dialectes bretons, s'en tiennent à la morphologie, au lexique ou à des considérations générales sur la prononciation. Mais rien sur la palatalisation. Bien que certains en parlent, il faut lire entre les lignes pour s'en faire une idée précise et découvrir qu'elle semblait d'une toute autre nature que celle qui s'entend aujourd'hui. Si des érudits si proches de nous dans le temps ne l'évoquent pas, ou en des termes si différents de notre propre expérience, peut-on admettre que le phénomène soit
\end{abstract}

\footnotetext{
* Maître de conférences de breton, CRBC (EA 4451-UMS 3554), UBO/ueb.

1. Formule qui ne semble pas très ancienne.
} 
si ancien qu'on l'a longtemps pensé ? Je me propose d'examiner la question, en mettant bien sûr en regard $1^{\prime} \mathrm{ALBB}^{2}$ avec le $\mathrm{NALBB}^{3}$ et l'ALRP ${ }^{4}$, mais surtout en pondérant l'apport de la géolinguistique, en recoupant les atlas par d'autres sources insuffisamment exploitées jusqu'ici. Je tâcherai de démontrer ainsi l'hypothèse d'une évolution très récente, prenant appui sur un fond très ancien. Le caractère dynamique et non-stabilisé du phénomène au moment même où il était décrit, ainsi que l'hyper-vernacularisation concomitante du breton expliqueraient en grande partie l'apparente irrationalité des données de terrain.

\section{Définitions}

En termes articulatoires, la palatalisation est le déplacement du point d'articulation d'une consonne ou d'une voyelle au niveau du palais (cf. schéma ci-dessous, illustrant quelques consonnes et la palatalisation $\mathrm{de} / \mathrm{k} /$ ).

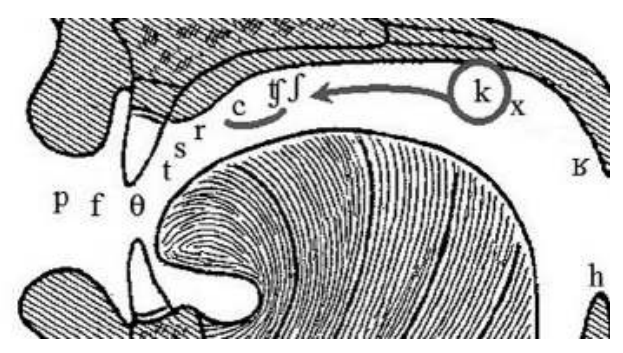

Tous les phonèmes peuvent en être affectés, même si dans les faits, cette évolution concerne surtout les articulations à proximité de la partie antérieure de la bouche. Quant à ce qui la provoque, on peut l'identifier comme une attraction articulatoire exercée par les voyelles. Pour prononcer un $[\mathrm{k}]$, le dos de la langue doit d'abord

2. Pierre Le Roux, Atlas linguistique de la Basse Bretagne, Rennes, Plihon et Homay et Paris, Champion, 1924-1963.

3. Jean LE DÛ (dir.), Nouvel atlas linguistique de la Basse Bretagne, Brest, CRBC, 2001.

4. Pascal Rolland, Atlas linguistique de la région de Pontivy, Brest, Ar skol vrezoneg, 1994. 
produire une occlusion au niveau du voile (velum) du palais. D'où le nom de consonne vélaire. Mais les organes anticipent toujours la voyelle suivante : la suite $[\mathrm{k}+\mathrm{o}]$ (voyelle postérieure) sera bien prononcée au fond de la cavité buccale ; en revanche, pour pouvoir émettre $[\mathrm{k}+\mathrm{i}]$ (voyelle antérieure), la zone de l'occlusion s'avance en direction de la région post-alvéolaire (derrière les dents). Dans le cas d'une palatalisation, la zone de contact est avancée à l'excès : elle se produit dans une région où l'occlusion du conduit buccal ne peut être complète. Elle laisse donc passer un souffle, une affriction plus ou moins nette : [ $\left.\mathrm{k}^{\prime}\right]$ ou $[\mathrm{c}]$ (assimilation progressive). Le phénomène inverse $[\mathrm{i}+\mathrm{k}]$ est également possible : la langue conserve alors la position du [i] au moment de prononcer le [k], d'où une friction [ic] (assimilation régressive). Susceptible de se phonologiser, la palatalisation commence généralement comme réalisation phonétique contingente, stade auquel elle est très inégale, peu prédictible selon les langues et selon les locuteurs.

\section{La palatalisation romane}

La palatalisation a souvent marqué des moments de basculement dans l'histoire des langues, en particulier des langues romanes, où elle sert de démarcation entre latin classique et latin tardif. Quasiment toute la Romania est ainsi affectée par une première palatalisation des vélaires devant $[\mathrm{i}-\mathrm{e}]$ aux $\mathrm{II}^{\mathrm{e}}-\mathrm{III} \mathrm{e}^{\mathrm{e}}$ siècles, qui fait passer un mot comme cera (cire) de ['ke冈ra]) à ['k'e冈ra], aboutissant à ['ce区ra] en proto-italien ou ['tse区ra] en gallo- et ibéro-roman. L'affriction se maintient jusqu'à nos jours en Italie : ['fe区ra], tandis qu'elle évoluera en consonne simple $/ \mathrm{s} /$ en français, espagnol, portugais etc.

Le français se distingue comme particulièrement sujet à la palatalisation. $\mathrm{Au} \mathrm{V} \mathrm{V}^{\mathrm{e}}$ siècle, il est touché par une deuxième vague, cette fois devant $[\mathrm{a}-\mathrm{o}]$, qui explique par exemple cattus $>$ chat, phénomène absent des autres langues romanes ${ }^{5}$. Le français classique en restera là. Le bon usage de la Cour, transmis à la langue des salons, à la langue scolaire du $\mathrm{XIX}^{\mathrm{e}}$ siècle et à la langue médiatique du $\mathrm{XX}^{\mathrm{e}}$ n'évoluera plus sur ce point. Mais le français populaire et rural connaîtra encore une troisième vague de palatalisation, ignorée de

5. Et en français, absent également des dialectes normands et picards. 
la langue savante : une nouvelle palatalisation des vélaires devant voyelles antérieures, qui tend à transformer par exemple quille, curé

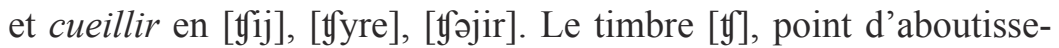
ment de l'évolution, est rare cependant : l'ALF montre des faits palatalisés à divers stades, saisis en pleine évolution. Karin Ringenson note cette instabilité fondamentale du phénomène ${ }^{6}$ et en s'appuyant sur $1^{\prime} \mathrm{ALF}^{7}$ elle retient sept degrés possibles de palatalisation pour le $/ \mathrm{k} /$ des patois français ${ }^{8}$. Elle démontre également que cette caractéristique affecte davantage les parlers de l'ouest de la France, comme le montre la carte de synthèse ci-dessous, dérivée de ses travaux :

Ci-contre : palatalisation de /k/ devant /i, y, $\mathrm{e}$, œ/ dans l'ALF. En rouge : / $/$ est palatalisé en présence des quatre voyelles ; en orange foncé, devant trois (plusieurs combinaisons possibles); en orange clair, devant deux ; en jaune, une seule. L'intensité du phénomène pourrait être corrélée

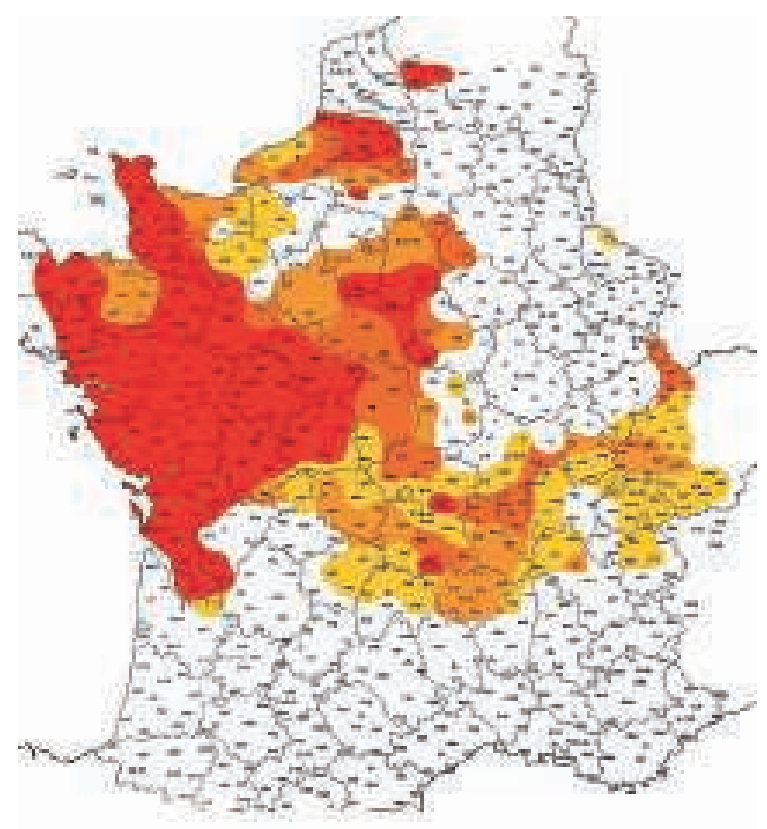
à son ancienneté.

Une autre corrélation apparaît aussi : ce sont les régions les plus exposées à une influence extérieure : latinité au sud et adstrat germanique au nord-est, qui sont les moins enclines à palataliser.

6. Karin Ringenson, La palatalisation de K devant une voyelle antérieure en français, Paris, Champion, 1922, p. 26.

7. Jules GilliÉron et Edmond Edmont, Atlas linguistique de la France, Paris, Champion, 1902-1910.

8. K. Ringenson, op. cit., p. 161. 
Les régions les plus touchées seraient donc au contraire, celles où le substrat gaulois serait le plus actif (hypothèse avancée d'ailleurs par Becker en 1947, cité sans mention de source par Fernand Carton ${ }^{9}$.

Dans la mesure où le français normé n'a pas été touché, au profit des seuls patois, cette palatalisation a laissé peu de traces à l'écrit, ce qui pose de grandes difficultés de datation. Elle est surtout attestée dans l'ouest à la fin du XVIIe et au début du XVIII ${ }^{e}$ siècle ${ }^{10}$, mais Ringenson n'hésite pas à la reculer au XVI ${ }^{\mathrm{e}}{ }^{11}$, tandis que Michaelsson en donne des attestations pour Paris dès $1292^{12}$.

Contrairement aux apparences, le français si uniforme d'aujourd'hui, est toujours travaillé par cette tendance lourde. Pour prendre des exemples facilement vérifiables sur internet, à l'audition des journaux télévisés de France 2, ma perception est qu'une bonne moitié des intervenants ont une prononciation très légèrement palatalisée des /k/ pour des mots comme qui, que, politique ou sécurité $^{13}$. Ce trait est généralement considéré comme un marqueur du parler populaire, qui indiquerait donc les origines sociales des journalistes. Cependant les faits sont distribués de façon complexe : le monde ouvrier en donne certes des exemples parfois très frappants ${ }^{14}$, mais on peut aussi l'entendre assez atténuée chez des personnalités aussi différentes que Josiane Balasko, Alain Finkielkraut ou Marion Maréchal-Le Pen. En revanche, je ne l'entends pas dans les enregistrements de la voix d'Henri Krasucki ou Bernard Thibault. Ces différences ne s'expliquent pas par des origines géographiques diver-

9. Fernand CARTON, Introduction à la phonétique du français, Paris, Bordas, 1974, p. 154.

10. Ferdinand BRUNOT, Histoire de la langue française des origines à nos jours, Paris, Armand Colin, 1966, p. 976.

11. K. RingENSON, op. cit., p. 107-110.

12. Karl MiChAELSSON, «Quelques variantes notées dans la prononciation parisienne au temps de Philippe le Bel », Atti dell'VIII Congresso internazionale di studi romanzi (vol. II), Florence, Sansoni, 1956. (Je remercie J.-P. Chauveau de m'avoir mis sur cette piste.)

13. La palatalisation est extrêmement prononcée chez Gérard Grizbec, où elle affecte même $/ \mathrm{k}+\mathrm{a} /$.

14. Cf. Jean-Pierre Mercier, délégué CGT à PSA Aulnay-sous-Bois, bien présent dans les médias. On l'entend aussi dans le parler de certains dockers du Havre diffusés dans La fabrique de l'histoire, France Culture, 11 mars 2014. 
gentes : toutes les personnes citées étant nées ou ayant été élevées depuis leur plus jeune âge dans un étroit périmètre autour de Paris.

Le français, par ailleurs, lie étroitement palatalisation des vélaires $/ \mathrm{k}-\mathrm{g} /$ et des dentales $/ \mathrm{t}-\mathrm{d} /$. Aux XVII e et XVIII ${ }^{\mathrm{e}}$ siècles, ces consonnes finissent souvent par se confondre dans l'usage populaire. D'où tabatière, remplaçant l'ancien tabaquière. Les paysans de la langue classique ${ }^{15}$, qui sont souvent les premières attestations du phénomène, disent ainsi Guieu et guiable, amiquié, etc. ${ }^{16}$. Ce type d'assimilation s'entend également aujourd'hui encore: dans les quartiers populaires à forte composante immigrée de la moitié nord de la France ${ }^{17}$ et quasi-universellement en français méridional, $/ \mathrm{t}+$ i/ tend plus ou moins vers [tçi] ou [tij] et des mots comme timide et quartier y deviennent presque 'tchimide' et 'quartchier' 18 .

De son côté, le français du nouveau monde, qui était porteur des mêmes traits, les a fait évoluer d'une manière spécifique. Chez la plupart des Québécois, le timbre des / $\mathrm{k}$ - g/ ne semble absolument pas palatal. Pourtant, la palatalisation des vélaires y aurait existé ${ }^{19}$, mais elle aurait fortement régressé assez récemment sous la pression de l'école ${ }^{20}$. La palatalisation concerne donc surtout les dentales, particulièrement sensibles en Acadie (cf. Acadien > Cajun), en Beauce

15. La Fontaine, La coupe enchantée, 1671; Molière, Don Juan, 1665 ; MARIVAUX, Les surprises de l'amour, 1722.

16. F. BRUNOT, op. cit., p. 976 ; K. RingENSON, op. cit., p. 88.

17. Lorenzo Devilla, et Cyril Trimaille, «Variantes palatalisées/affriquées en français hexagonal : quel(s) statut(s) sociolinguistique(s) pour quel destin ?», Actes du XXVe congrès international de linguistique et philologie romanes, Innsbruck, 2007.

18. Il me semble donc (mais mes compétences en ce domaine sont très limitées) que les palatalisations en français actuel opposent les locuteurs selon des logiques de groupes, où l'âge interfère avec les déterminants sociaux : chez les Parisiens de plus de 40 ans, [k'] serait le signe d'une origine populaire, que certains férus de culture parviendraient à gommer totalement. Pour les jeunes Parisiens, c'est au contraire plutôt dans les quartiers favorisés (ce qui explique la présence de M. Maréchal-Le Pen dans la liste précédente) que l'on palatalise fréquemment [ $\left.k^{\prime}\right]$ et que l'on fait subir à $/ t /+/ i$ / une légère assibilation. Dans les banlieues, les jeunes font surtout entendre la palatalisation de $/ \mathrm{t} / \mathrm{t} / \mathrm{i} / \mathrm{en}$ [tç].

19. Cette prononciation, considérée comme populaire, est attestée par Gendron, cité par Adèle SAINT PIERRE, « Le phénomène de la palatalisation au Québec : étude historique et phonétique », mémoire de Master, University of Maine, 2002 p. 30.

20. Ibid., p. 31-32. 
québécoise et dans le Maine (USA), ainsi que dans l'assibilation $\left[\mathrm{t}^{\mathrm{s}}\right]-\left[\mathrm{d}^{\mathrm{Z}}\right]$ caractéristique du français québécois, qui serait le vestige de la dépalatalisation $\mathrm{de} / \mathrm{t}-\mathrm{d} /{ }^{21}$.

\section{La palatalisation en breton}

La première description scientifique et cartographique rigoureuse du phénomène en breton est, à ma connaissance, due à F. Falc'hun, dans son Histoire de la langue bretonne d'après la géographie linguistique. À partir de 22 cartes illustrant la palatalisation de $/ \mathrm{k} /$, il montre une nette coupure (matérialisée par la ligne a) entre une moitié sud-est du domaine bretonnant, où elle est très présente, et une moitié nord-ouest, où elle est quasiment inconnue ${ }^{22}$.

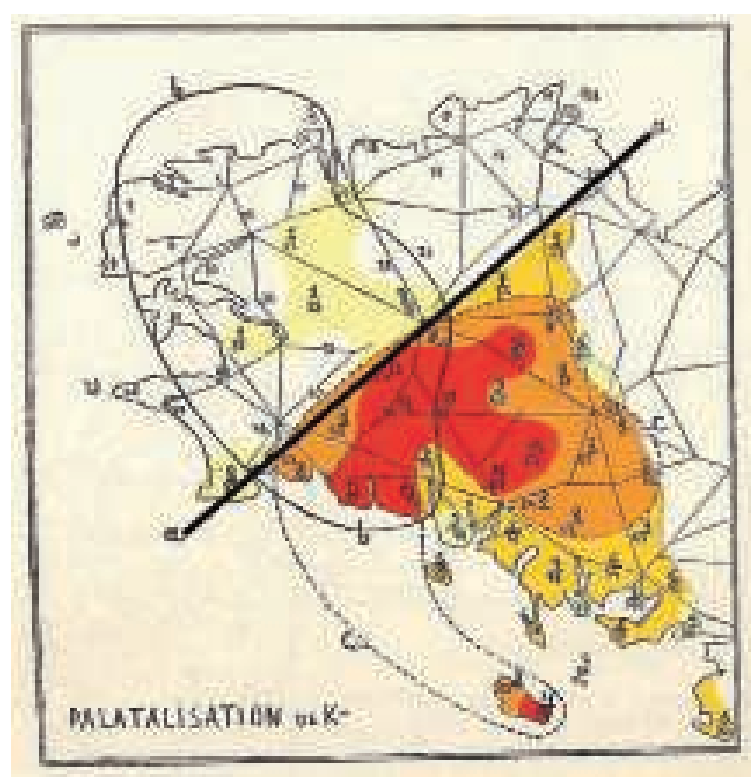

21. Ibid., p. 35, 50 et sq. Les diverses hypothèses concernant l'assibilation québécoise sont discutées de façon détaillée par Yves-Charles MORIN, « Les premiers immigrants et la prononciation du français au Québec », Revue québécoise de linguistique, $\mathrm{n}^{\circ} 31-1,2002$, en particulier p. 62-67.

22. François FALC'HUN, Histoire de la langue bretonne d'après la géographie linguistique, Paris, PUF, 1963, p. 187. 
Cette carte (ici colorisée) va fixer pour plusieurs décennies la représentation de la palatalisation pour les locuteurs érudits : forte prévalence en vannetais, avec domination du bas-vannetais ; importante en Cornouaille également ${ }^{23}$; moindre occurrence près des côtes que dans les terres.

Pourtant, elle est loin de rendre compte à elle seule de la palatalisation, puisqu'elle ne présente que les / $\mathrm{k} /$ initiaux. Pour avoir une vision complète du phénomène, il faut élargir le champ d'observation. Je me suis donc employé dans un premier temps à refaire, en quelque sorte, le travail de Falc'hun, en le systématisant : en relevant sur les cartes de l'ALBB toutes les occurrences de palatalisations appartenant à un type « vannetais».

\section{Les phénomènes écartés}

Il faut cependant signaler que la palatalisation affecte une gamme de phonèmes beaucoup plus large que les seuls $/ \mathrm{k}-\mathrm{g} /$ et secondement, que ceci s'observe à travers tout le domaine bretonnant Pour établir un type de palatalisation « vannetais », il faut donc sélectionner un nombre limité de phénomènes : ceux qui présentent une distribution géographique et une concentration spatiale axée sur un grand tiers sud-est de la Basse-Bretagne. Seront par contre exclus, ceux qui ne présentent pas le même caractère structurant en termes géolinguistiques. Soit parce que très localisés, soit au contraire très largement diffusés :

- La palatalisation de /t/ et /d/. Cette tendance est attestée en breton de deux manières. Soit à l'état de traces : dans la région de Pontivy, par exemple, certains /d/ étymologiques deviennent une consonne intermédiaire entre $[\mathrm{d}]$ et $[\mathrm{g}]^{24}$. De même, en bas-vannetais, tuemm (chaud) est souvent réalisé $[\mathrm{com}]^{25}$. Deuxième tendance, l'évolution en $\left[\int, 3\right]$ des pluriels des mots se terminant en $/ \mathrm{t}, \mathrm{d} / \mathrm{koad}$

23. La palatalisation cornouaillaise est moins ressentie, probablement du fait de son articulation dorso-palatale, plus discrète (cf. infra).

24. ALBB, c. 6, pt. $61:<\mathrm{g}^{\mathrm{d}} \rightsquigarrow \mathrm{h} \rrbracket \mathrm{n} \rrbracket \mathrm{m} \bigotimes>$ pour di-ac'hann-mañ, d'ici. Voir aussi ALRP, c. 32 et E. Thibault, « Notes sur le parler breton de Cléguérec (Morbihan) », Revue celtique, t. 35, 1914, p. 19.

25. ALRP, c. 134. 
(bois) $>$ koajoù ; hent (route) $>$ henchoù ${ }^{26}$. Celle-ci est non seulement très large, mais elle est en outre attestée exclusivement hors du pays vannetais, en parfait négatif de celui-ci.

- Le groupe /ik/. Là aussi, le phénomène est extrêmement répandu, particulièrement en finale ${ }^{27}$. En position médiane, il est aussi très courant dans le Léon ${ }^{28}$.

- Le /g/ post-tonique évolué en [j]. Celui-ci semble continuer une tendance plus ancienne, attestée par l'évolution de argant (argent) > arc'hant, gallois arian; ou le pluriel des mots en $-e g+$ ien $>$-eien (beleg $>$ beleien, prêtre-s). Ce type de palatalisation est aussi très répandu ${ }^{29}$, mais son caractère post-tonique limite ou exclut toute occurrence dans de larges régions du sud-est, à accent final.

- Les fricatives. Plusieurs types entrent dans cette catégorie :

- Le /h/ réalisé [ç], phénomène trop local (essentiellement basvannetais et marges cornouaillaises contiguës).

- Les $/ n /$ et $/ 1 /$, réalisées $[n]$ et $[K]$, qui concernent à peu près tous les parlers.

- Les $/ \mathrm{s}$ / évolués en $/ \mathrm{J} /$, phénomènes également très répandus.

- Les voyelles. C'est par exemple le cas dans pemp (cinq), qui évolue localement vers des types $[\mathrm{pjəm}]^{30}$. Je préfère analyser ces évolutions vocaliques comme diphtongaisons plutôt que comme palatalisations.

\section{Les palatalisations examinées}

Une fois dégagées des évolutions touchant la langue dans son ensemble et celles qui relèvent de l'hyper-localisme, trois grands phénomènes vont être observés :

- Les vélaires /k/ et /g/ devant /i, e, y, œ, ə/. La palatalisation de ces consonnes est une tendance très répandue, quoique nonsystématique. Le phénomène paraît bien territorialisé dans les limites définies par Falc'hun, c'est-à-dire bien au-delà du seul

26. ALBB, c. 380 et 317.

27. ALBB, c. $386, k i g$, viande.

28. François FAlC'HUn, Le système consonantique du breton, Rennes, Plihon, 1951, p. 27-29.

29. NALBB, c. 73, ugent, vingt et 314 , karrigell, brouette.

30. ALBB, c. 122. 
pays vannetais, mais avec semble-t-il une plus grande régularité à l'intérieur de celui-ci. En domaine vannetais stricto-sensu, elle atteint généralement l'affriction apico-palatale [t] et [d]], mais il est possible d'entendre (surtout dans les régions contigües) des dorso-palatales [c] et [f] (cf. infra). Il n'existe que deux exemples, très localisés de la même palatalisation devant /a/ et /u/ : /, byga:'le/, enfants, et/,di'gur/, ouvert ${ }^{31}$.

Il est clair cependant qu'une telle évolution linguistique ne se produit jamais seule : elle est le produit d'une inflexion articulatoire qui a forcément d'autre effets, et elle produit à son tour des effets collatéraux. Ceux-ci vont se manifester ainsi :

- Le groupe /sk/ devant voyelle antérieure ou centrale. La palatalisation de $/ \mathrm{k} /$ en pareil contexte entraine une prononciation [sk'], [sc] ou [st']. Si certaines langues permettent une telle distribution, les bretonnants s'y sont montrés rétifs ${ }^{32}$. D'où une évolution vers [ $]$ ].

- Les groupes /st/ et /sp/ ; /sk/ devant voyelle postérieure. La même évolution que précédemment est attestée pour/st/ en moyenbreton : avec [t'] palatal dans l'emprunt à l'ancien-français mestier (métier), /st/ donne [S] > micher. Mais il s'agit d'un exemple isolé, qui confirme la marginalité de [ $\left.\mathrm{t}^{\prime}\right]$ palatal dans le système articulatoire breton. L'évolution vannetaise aura consisté à changer tous les /st/ en $\left[\int \mathrm{t}\right]^{33}$. C'est probablement par analogie que le groupe /sp/ a subi la même évolution vers $\left[\int \mathrm{p}\right]$. De façon très localisée, sur une frange contiguë à la frontière linguistique, le groupe /sk/ devient également [ $\mathrm{k}]$ devant voyelle postérieure ${ }^{34}$.

31. ALBB, c. 44 et 152.

32. La comparaison entre ALBB et NALBB montre une régression rapide de ces prononciations transitoires.

33. On décèle une tendance analogue, bien que moins nette et moins systématique sur la côte léonarde.

34. Traitement étendu devant la pause : kouske (dormait) peut être réalisé [ku'Jke], mais en principe, jamais $*[\mathrm{ku}\} \mathrm{e}]$, car le mot est traversé par une frontière entre deux monèmes : base verbale/kousk-/ et marque morphologique/-e/. Le blocage morphologique l'emporte sur la tendance phonétique. Gwiskiñ (habiller) devrait en principe se comporter de même, pourtant, à Belle-Ile, le /sk/ devient [ $\mathrm{C}$ ] (ALBB : c. 303). 


\section{Extension}

Par économie, j'ai limité mon observation aux régions situées au sud-est de la ligne a de Falc'hun, ainsi qu'aux premiers points de l'ALBB au-delà de cette ligne. En cumulant les phénomènes retenus, ce sont quatre-vingt-huit cartes ${ }^{35}$ qui permettent d'obtenir la carte de synthèse suivante ${ }^{36}$ :

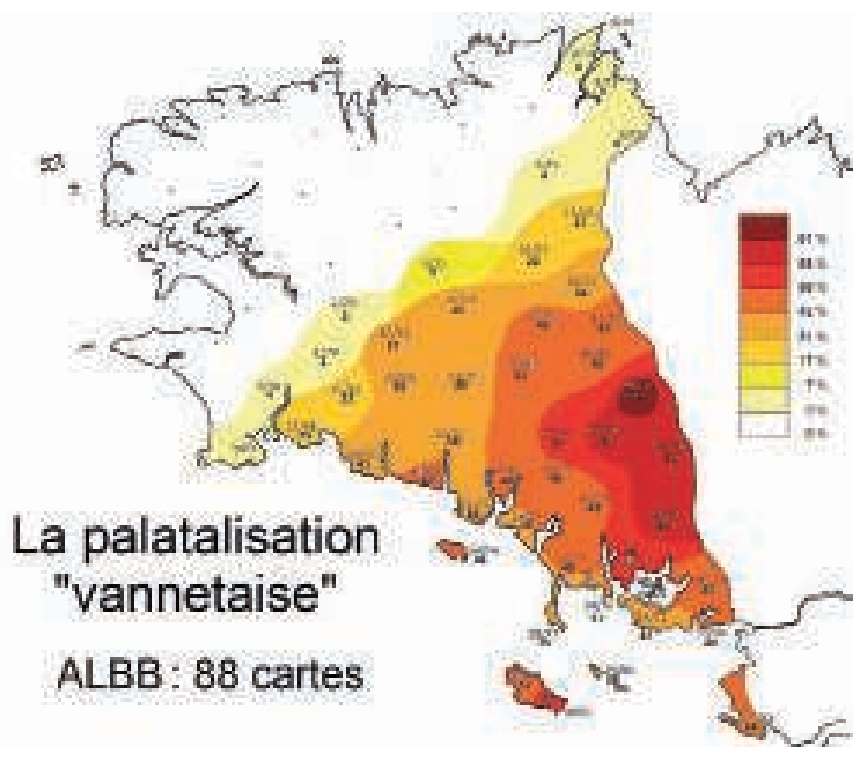

35. Pour /k/, cartes : 114, 206, 235, 242, 250, 361, 362, 364, 366, 367, 368, 369, 370, $371,372,373,374,375,376,377,378,379,381,386,443,511$; pour /g/ et / gч-/, cartes : 44, 136, 152, 178, 201, 208, 210, 211, 212, 213, 214, 219, 222, 223, $363,366,367,368,388,461,472,514$; 144, 255, 287, 288, 290, 291, 292, 293 , 294, 295, 296, 298, 299, 300, 303, 304, 467 ; pour /sk - st - sp/, cartes : 104, 150, $161,170,185,260,303,441,522,523,557,565,566,567,568,569 ; 414,566$, 569 ; 162. Certaines cartes ont été prises en compte deux fois : la carte 300 pour gwinizh et gwinizh-du par exemple.

36. Le NALBB s'est révélé plus délicat à manier. D'une part parce que les données exploitables sont relativement peu nombreuses : 12 cartes pour $/ \mathrm{g} /$ contre 22 dans l'ALBB ; 7 cartes pour /gu-/ contre 18 . Ceci pose un possible problème d'adéquation à l'échelle : le réseau du NALBB étant très dense, construire des cartes cumulatives suppose un très grand nombre de données pour produire des ensembles lisibles et sortir de l'" effet mosaïque ». Il existe par ailleurs un fort déséquilibre entre les phénomènes observées $(/ \mathrm{k}-\mathrm{g}-\mathrm{st}-\mathrm{sk} . . /)$, alors que ceuxci sont en nombre à peu près équivalents dans l'ALBB. 
Par rapport à la carte de Falc'hun, on notera un net recentrage du phénomène vers l'est. Alors que l'ancien pays de Vannes semblait se diluer à l'examen des seuls $/ \mathrm{k} /$, il émerge cette fois très nettement, avec régulièrement plus de $42 \%$ d'occurrences palatalisées, quand les parlers cornouaillais les plus palatalisants se situent plutôt dans un étiage d'un quart à un tiers. En revanche, la côte morbihannaise est effectivement moins concernée que l'intérieur des terres. Ceci est la conséquence d'une diffusion radiale, à partir d'un épicentre situé dans le nord du haut-vannetais (pt. ALBB 63 : Noyal-Pontivy : $61 \%$ ). Une telle distribution, outre qu'elle permet de souligner la prégnance des parlers vannetais dans le développement de cette tendance, tendrait à prouver par sa régularité la pertinence à associer les trois phénomènes retenus.

\section{Dynamique}

\section{Des origines lointaines?}

Devant cette localisation, dans le prolongement de la Bretagne romane, ajoutée à la parenté phonétique entre palatalisation bretonne et français dialectal de l'ouest, il est tentant de supposer un lien de continuité, par-delà la différence de langues. Le quidam le fait sans état d'âme, les chercheurs sont plus nuancés. Falc'hun, mettant en corrélation prévalence de la palatalisation et distribution des toponymes (commençant par Plou- ou terminés par - ac), avance que l'absence de palatalisation « continue les traditions phonétiques (...) des immigrés bretons d'origines insulaires, et la palatalisation du $k$ les habitudes articulatoires des populations de l'Armorique ${ }^{37} \gg$. S'il reste prudent, en ne proposant pas de date pour l'évolution, son hypothèse sous-jacente relie la palatalisation à l'influence d'un substrat gaulois. Fleuriot adopte le même schéma, mais en changeant les rôles. Pour lui, le substrat en cause est roman $^{38}$ : puisque la palatalisation de $/ \mathrm{k} /$ est inconnue ou négligeable en domaine celtique hors du sudest breton; puisque seule la première palatalisation de type roman (devant /e, i/) est attestée en breton, et non la seconde (devant/a, o/),

37. F. FALC'HUN, op. cit. (1963), p. 187.

38. Léon Fleuriot, Les origines de la Bretagne, Paris, Payot, 1980, p. 68-69 et 8687. 
c'est que la région a changé de langue entre ces deux évolutions. Les populations du vannetais auraient donc été romanophones jusqu'au $\mathrm{V}^{\mathrm{e}}$ siècle ${ }^{39}$, puis la grande migration bretonne les aurait (re)celtisées. Mais elles auraient conservé leur accent roman, qui les faisait déjà palataliser devant /e, i/. Le changement linguistique aurait ensuite préservé le breton de l'évolution romane ultérieure. Le point commun de ces deux chercheurs est en tout cas de faire remonter la palatalisation à une très haute époque : il s'agirait d'un phénomène originel, remontant aux premiers peuplements bretons.

Jackson ne croit pas à une telle hypothèse. D'abord parce que pour lui, il n'y a pas de continuité entre gaulois et breton, qui n'est pas autre chose que du brittonique ${ }^{40}$. Il n'y a donc pas lieu de se référer à une source extérieure et il ne faut pas exclure a priori un développement purement autonome de la palatalisation ${ }^{41}$. Et de toute façon, si influence extérieure il y a, elle ne vient pas selon lui du latin tardif, mais de l'ancien français ${ }^{42}$. Celle-ci aurait donc été très significative, puisqu'il la juge suffisamment forte vers l'an mil pour avoir pu bloquer en vannetais l'évolution de l'accent ${ }^{43}$. Mais il écarte fermement une évolution aussi précoce que celle envisagée par Falc'hun ${ }^{44}$, au motif que les faits linguistiques aussi anciens sont trop mal connus. En privilégiant la documentation disponible : enquêtes de terrain, ALBB et quelques sources imprimées, il réalise une analyse serrée des voyelles ${ }^{45}$ qui conclut à l'apparition de la palatalisation entre les $\mathrm{XI}^{\mathrm{e}}-\mathrm{XII}{ }^{\mathrm{e}}$ siècles au plus tôt et le $\mathrm{XV}^{\mathrm{e}}$ siècle au plus tard. Raude recule même encore cette limite ${ }^{46}$. En exploitant

39. Fleuriot se sert d'une échelle temporelle plus large. Je me fie à celle utilisée par les romanistes (F. CARTON, op. cit. ; Geneviève Joly, Précis de phonétique historique du français, Paris, Armand Colin, 1995 ; Annick EngleberT, Introduction à la phonétique historique du français, Bruxelles, De Boeck, 2009).

40. Kenneth JACKSON, A Historical Phonology of Breton, Dublin, The Dublin Institute for Advanced Studies, 1967, p. 31-32.

41. Ibid., p. 403.

42. Ibid., p. 29.

43. Ibid., p. 83.

44. Ibid., p. 29-30.

45. Ibid., p. 401-402.

46. Alain RAUDE, « La palatalisation des consonnes vélaires en breton et en brittoroman », La Bretagne linguistique, n 5, Brest, CRBC, 1989. 
d'autres cartes de l'ALBB, qu'il met en relation avec diverses sources écrites et en s'appuyant sur sa propre expérience de locuteur, il date l'origine de la palatalisation au XVIII ${ }^{\mathrm{e}}$ siècle et la décrit comme un mouvement toujours en cours au $\mathrm{XX}^{\mathrm{e}}$. Mais il remet en avant l'hypothèse d'une origine française : pour lui, la palatalisation a été introduite par Corlay depuis les parlers gallos de Haute-Bretagne, et se serait ensuite propagée vers le sud-ouest, affectant donc basvannetais et Cornouaille de l'est, mais laissant de côté le vannetais intérieur.

Chacune de ces hypothèses présente un certain nombre de faiblesses. Jackson a abondamment critiqué la théorie de Falc'hun, en lui reprochant de faire des rapprochements spéculatifs, en l'absence de documentation. Ce qui est peut-être juste, mais c'est le propre des explications par substrat de ne guère se démontrer qu'au moyen de cartes et en mettant en relation l'archéologie, l'onomastique, aujourd'hui la génétique etc. au service de la linguistique. S'il est vrai que l'hypothèse falc'hunienne est peu vérifiable, elle est du moins cohérente et recoupée par la multiplicité des approches. Fleuriot présente de son côté une singulière contradiction : si le changement de langue laisse des traces dans un sens, il en laisse aussi forcément dans l'autre. Donc, avant l'«archéo-vannetais» brittonique à accent roman supposé par Fleuriot, le «paléo-vannetais » roman qui le précéda fut nécessairement du roman avec un accent gaulois. Par conséquent la palatalisation qui s'y est développée n'est pas forcément un legs roman, mais peut-être bien celtique continental (comme le laisse penser la carte d'après Ringenson supra). Pour convaincante que soit la démonstration de linguistique historique de Jackson, son rejet de l'ancienneté de la palatalisation paraît également contradictoire avec l'affirmation d'une influence française précoce : à supposer que celle-ci ait été assez puissante pour agir sur le système accentuel (ce qui me paraît très douteux), comment écarter dans un même mouvement qu'elle ait pu transmettre aussi des innovations articulatoires et reconfigurer le système phonologique ? Et si la palatalisation s'est développée de façon autonome, le hasard a curieusement tout fait pour induire le linguiste en erreur, puisqu'elle est localisée dans la partie la plus romanisée de la Basse-Bretagne... Quant à A. Raude, les cartes qu'il avance pour affirmer l'existence 
d'une aire de diffusion à partir de Corlay sont peu convaincantes ${ }^{47}$. Ses données sont en outre fort peu nombreuses et surtout, elles ont le tort de traiter à égalité le plan superficiel du lexique (où les diffusions sont rapides et faciles) et le soubassement profond auquel appartient la phonologie (qui n'adopte les innovations extérieures que dans le cas d'une déstabilisation profonde du système originel, résultant d'un bilinguisme asymétrique à grande échelle ${ }^{48}$ ). Or, si le breton connaît bien une pression de cet ordre aujourd'hui venant du français, il ne pouvait en être ainsi aux XVIII e ${ }^{\mathrm{e}}$ XIX ${ }^{\mathrm{e}}$ siècles, quand le français était une langue étrangère pour une vaste majorité de Bas-Bretons.

Ce qui frappe en tout cas, c'est l'opposition entre les méthodes de datation : plus la démonstration repose sur les cartes (Falc'hun et Fleuriot), plus les auteurs privilégient une date ancienne. À l'inverse, plus l'on s'appuie sur des sources écrites, plus s'impose l'idée d'une date récente.

\section{Évolutions récentes}

Avant d'examiner ces sources écrites, il vaut la peine de se pencher sur d'autres cartes, dont ne disposaient pas les chercheurs

47. Même si la carte 20 de l'ALBB montre effectivement une aire centrée sur l'axe Rostrenen-Corlay, le sens de diffusion n'est pas évident. D'autres cartes qu'il invoque me laissent encore plus perplexe. Son exemple pour la carte 564 est par ailleurs erroné : skabiaw est attesté à Mûr et non à Corlay.

48. On peut le mesurer en comparant les français de France, du Québec et de Louisiane. Soumis à une forte pression, le français louisianais est probablement en voie de perdre son phonétisme propre : l'alignement sur l'anglais s'entend ainsi nettement dans le /r/, le plus souvent rétroflexe. En revanche, l'anglais reste impuissant à influencer la phonétique du français québécois, qui généralement montre davantage de proximité avec les français dialectaux. Ceci bien que les Québécois prononcent les emprunts anglais avec un fort accent américain. Beaucoup plus détachés de l'anglais, les Français, quant à eux, plient sans vergogne ces emprunts à la phonologie hexagonale : à moins d'être professeur d'anglais ou assez pédant, personne en France ne prononce le $<$ th $>$ de Thatcher autrement que comme un /t/ ou un /s/. Idem pour la palatalisation : le $<\mathrm{ch}>$ de chewing-gum est réalisé $/ \mathrm{g} /$ et non $/ \mathfrak{g} /$. Les emprunts plus récents comme check up présentent bien une affriction, du fait d'une plus grande familiarité avec l'anglais scolaire dans les générations qui l'ont emprunté, mais l'imitation superficielle ou l'emprunt ponctuel ne présument pas de la stabilisation à long terme de cette articulation, encore moins qu'elle puisse influencer de l'intérieur les sons autochtones du français. 
précédemment mentionnés, celles du NALBB. Les enquêtes qui en sont à l'origine ont été réalisées entre 1969 et 1995, soit auprès d'informateurs en décalage d'une à deux générations par rapport à l'ALBB. Il existe 27 cartes exploitables communes aux deux atlas ${ }^{49}$. Aucune ne montre une régression de la palatalisation. Le tableau suivant met en regard les deux enquêtes :

\begin{tabular}{|c|c|c|c|c|}
\hline & Mots & ALBB & NALBB & Phonème(s) \\
\hline \multirow[t]{12}{*}{ Identité : 12} & Ken- & 375 & 138 & \multirow[t]{4}{*}{$/ \mathrm{k} /$} \\
\hline & Kerzhet & 379 & 449 & \\
\hline & Ket & $\begin{array}{l}206,235, \\
242,250\end{array}$ & 29,30 & \\
\hline & Kezeg & 443 & 327 & \\
\hline & Givri & 219 & 370 & \multirow[t]{5}{*}{$/ \mathrm{g} /$} \\
\hline & Kegi & 388 & 374 & \\
\hline & Bugale & 44 & 587 & \\
\hline & Karrigell & 363 & 314 & \\
\hline & Ugent & 136 & 73 & \\
\hline & Diskenn & 161 & 540 & \multirow[t]{3}{*}{$/ \mathrm{sk} /$} \\
\hline & Pesked & 522 & 524 & \\
\hline & Skalier & 104 & 539 & \\
\hline \multirow[t]{8}{*}{ Légère progression : 8} & Kêr & 366 & 10 & \multirow[t]{3}{*}{$/ \mathrm{k} /$} \\
\hline & Kirri & 362 & 313 & \\
\hline & Kizhier & 364 & 253 & \\
\hline & Digor & 152 & 544 & $/ \mathrm{g} /$ \\
\hline & Pesk & 522 & 523 & \multirow{3}{*}{$/ \mathrm{sk} /$} \\
\hline & Skuizh & 569 & 445 & \\
\hline & Pesketaer & 523 & 521 & \\
\hline & Kreisteiz & 414 & 99 & $/ \mathrm{st} /$ \\
\hline \multirow[t]{6}{*}{ Forte progression : 6} & Kelien & 371 & 242 & \multirow[t]{2}{*}{$/ \mathrm{k} /$} \\
\hline & Kig & 386 & 518 & \\
\hline & Pegement & 514 & 36 & /g/ \\
\hline & Gwenan & 291 & 246 & \multirow[t]{3}{*}{ 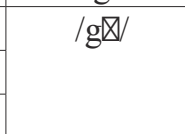 } \\
\hline & Gwener & 144 & 119 & \\
\hline & Gwinizh & 300 & 320 & \\
\hline Très forte progression : 1 & Gwez & 298 & 172 & 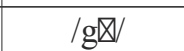 \\
\hline
\end{tabular}

49. Une carte est jugée inexploitable quand elle présente trop de formes mutées par exemple. 
Il est difficile de trouver une logique prédictive dans cette liste : la palatalisation semble progresser, en terme structuraux, de façon aléatoire et, sur le terrain, assez anarchique. De vastes zones palatalisent pour un terme, mais pas pour un autre, sans que l'on puisse avancer une explication claire (distribution, étymologie, etc.).

Un fait particulièrement remarquable est toutefois l'évolution $\mathrm{du}$ groupe /gu-/ initial. Alors que sa palatalisation est rare en 1911-1912 $2^{50}$, elle est devenue quasi systématique dans les parlers d'aujourd'hui. L'évolution la plus spectaculaire revenant aux cartes gwez, arbres : seuls Locmaria, en Belle-île et Houat palatalisent dans l'ALBB, contre environ un tiers de la Basse-Bretagne dans le NALBB. Le caractère impressionniste de la notation (il n'existe que deux degrés de palatalisation : $\langle\bigotimes\rangle$ et $\left\langle^{2}\right\rangle$ dans l'ALBB et un seul : $<\mathrm{c}>$ dans le NALBB, avec les voisées correspondantes) limite la visibilité du phénomène ${ }^{51}$, mais il est clair que la palatalisation de /gu-/ est une innovation du $\mathrm{XX}^{\mathrm{e}}$ siècle.

\section{Les sources écrites}

Le recours préférentiel aux cartes des atlas plutôt qu'aux documents écrits pour faire l'histoire de la langue est un réflexe inconditionnel pour nombre de linguistes s'intéressant au breton. Probablement parce que les documents y sont moins nombreux que pour les langues de large diffusion. Sans doute aussi en raison de la qualité même des outils qui se révèlent une mine inépuisable et du travail d'interprétation de Falc'hun, à l'époque novateur, qui a permis de révolutionner les hypothèses antérieures. L'utilisation des cartes ne doit pourtant pas conduire à négliger un corpus écrit, qui reste largement exploitable.

S'agissant de la palatalisation, il est cependant bien connu que le principal dialecte concerné, le vannetais (à prendre ici comme la forme livresque usitée dans l'ancien pays de Vannes) n'a jamais

50. ALBB 287, gwelet, voir ; 290, gwenn, blanc ; 292, Gwened, Vannes etc.

51. Comme signalé plus haut, l'ALF distingue sept degrés. Il paraît douteux que la palatalisation de givri (chèvres) notée dans le NALBB < <i'vre> à Pluneret (pt.178) soit en termes articulatoires exactement la même qu'à Roudouallec (pt. 85) : $<^{\prime}$ Jeuri $>$ ou que celle présente dans digeriñ (ouvrir) : $<$ di'łe:ri $>$ à SaintPabu (pt.8). 
développé de graphème spécifique pour la noter de façon systématique et univoque. Par parenthèse, on notera que, même en arguant du retard habituel de l'écrit sur l'oral, cette absence de graphémisation constitue un argument supplémentaire pour une apparition récente de la palatalisation. Celle-ci doit donc être décelée en empruntant deux canaux : celui des descriptions explicites du breton et celui des graphies phonétiques. Deux tendances apparaissent assez nettement, suivant qu'elles mentionnent ou non la palatalisation. Chronologiquement, ces deux sous-corpus sont assez imbriqués, même si l'on note une légère antériorité de l'un par rapport à l'autre.

\section{Pas de palatalisation}

Les descriptions explicites du breton comprennent des notes de linguistes et de lexicographes, des plus amateurs aux plus chevronnés, qui ont eu l'occasion d'entendre du breton à des dates plus ou moins anciennes et d'en noter quelques caractéristiques.

a) En 1659, Julien Maunoir, dans l'introduction de son dictionnaire ${ }^{52}$ indique pour la première fois qu'il existe quatre dialectes bretons, mais n'en fournit aucun trait.

b) En 1723, Pierre de Châlons, dans le premier dictionnaire vannetais, donne quelques exemples de variation phonétique ${ }^{53}$, mais ne mentionne rien sur la palatalisation (à un détail près, sur lequel je reviendrai).

c) En 1744, Cillart de Kerampoul, dans son dictionnaire également, s'étend longuement sur les différences accentuelles entre breton de Vannes et d'ailleurs ${ }^{54}$. Il donne aussi des exemples de bon et de mauvais usage. Mais aucune allusion encore à la palatalisation.

d) En 1752, la préface du dictionnaire imprimé de Le Pelletier (manuscrit de 1716) donne des règles précises de correspondances phonétiques entre breton vannetais et les autres parlers ${ }^{55}$. Mais toujours rien sur la palatalisation.

52. Julien MAUnOIR, Le sacré Collège de Jésus, Quimper, Jean Hardouin, 1659, p. 19.

53. Pierre DE CHÂLONS, Dictionnaire breton-françois du diocèse de Vannes, Vannes, Heucqueville, 1723, p. II.

54. Claude-Vincent CillarT DE KerAmpoul, Dictionnaire françois-breton ou françois-celtique, Leyde, Compagnie de Jésus, 1744, p. V-VII.

55. Dom Louis Le Pelletier, Dictionnaire de la langue bretonne, Paris, Delaguette, 1752, p. 12-14. 
e) En 1836, la grammaire de Guillôme indique que « toutes les consonnes se prononcent comme dans le latin ${ }^{56} »$ (avec toutefois une petite entorse qui sera détaillée plus bas).

f) En 1902 encore, la grammaire de Guillevic et Le Goff explique que $\mathrm{G}$ et $\mathrm{K}$ « se prononcent comme en français » et ne prévoit pas de cas particuliers ${ }^{57}$.

Les graphies phonétiques ne laissent longtemps rien transparaître non plus :

g) En 1499, Arnold von Harff nous fournit le plus ancien témoignage. Voyageur allemand de passage à Nantes, il donne une liste de mots bretons, notés selon les normes graphiques de l'allemand rhénan médiéval. L'attribution du breton de von Harff à la région vannetaise est loin d'être évidente, malgré le sentiment de Ch. Guyonvarc'h, qui l'a étudié en détail ${ }^{58}$ et qui s'appuie notamment... sur des correspondances avec l'ALBB ${ }^{59}$. En tout cas, la graphie de von Harff, qui reste assez approximative, ne laisse transparaître aucune palatalisation pour les mots: kick (viande),

56. Joachim GuILLÔME, Grammaire française-bretonne, Vannes, Galles, 1836, p. 7.

57. Augustin GuilleviC et Pierre Le Goff, Grammaire bretonne du dialecte de Vannes, Vannes, Lafolye, 1902, p. 4. Ce silence dissimule sans doute une intention prescriptive. Dans son manuel de 1979 (Le breton parlé, Vannes, Bleun Brug), Meriadeg Herrieu ne peut plus ignorer ainsi la palatalisation, qui a connu entretemps un important développement. Mais il conseille de «prononcer comme c'est écrit » (p. 24). Il faudrait même refuser certaines prononciations et limiter au minimum les palatalisations (ibid., p. 22), parce que «c'est plus élégant » (Le breton du Morbihan, 2e édition du même ouvrage Vannes, Bleun Brug, 1981, p. 7).

58. Christian GuYONVARC'H, Aux origines du breton, le glossaire vannetais $d u$ chevalier Arnold von Harff, Rennes, Ogam-Celticum, 1984.

59. Dans la mesure où l'on ne sait rien du contexte du recueil, tout est imaginable : le chevalier a-t-il interrogé un seul informateur ou plusieurs ? A-t-il noté son breton à Nantes, où parmi les marins (et d'où venaient-ils ?) qui manœuvraient le bateau sur lequel il avait embarqué à Bordeaux ? Cet aristocrate de haut rang, de retour d'une mission diplomatique auprès du Pape, a-t-il interrogé les matelots ou le capitaine ? Un colporteur, son aubergiste ou un voyageur de belle allure ? Lui a-t-on livré du breton « local » ou une forme "koïnisée » ? Même s'il y a d'indéniables points communs entre certains termes de ce breton et celui décrit à Guérande à la fin du XIX ${ }^{\mathrm{e}}$ siècle, il est très hasardeux de privilégier la continuité plutôt que la possible multiplicité ou la polynomie des sources, et de le présenter comme un échantillon de breton guérandais du XVe siècle. 
ker (avoine), gwin (vin), gwinagere (vinaigre), pesket (poisson, avec confusion singulier/pluriel), ammestres (hôte, avec confusion masculin/féminin), amnestisses (hôtesse). Seul le /n/ est palatalisé dans narinck (non) et oinge (poule, en réalité oiseau).

h) Le premier texte identifiable comme du vannetais, le formulaire de prône de $1631^{60}$, note les vélaires en empruntant les normes graphiques du français. Là où le $<\mathrm{k}>$ est employé aujourd'hui, c'est donc $<\mathrm{c}>$ ou $<\mathrm{qu}>$ (ce dernier à l'initiale devant $e$ et $i$ ) qui apparaissent à l'époque. Rien ne permet de supposer une prononciation palatale dans les mots orthographiés ainsi.

i) En 1723 et 1744, il en va de même dans les dictionnaires de Châlons et de Cillart de Kerampoul. Celui de Châlons ${ }^{61}$ (breton-français) n'a pas d'entrée $\mathrm{K}$, et renvoie donc le lecteur à C ou à Q. Preuve que ces trois lettres notent le même son, un même mot peut s'écrire avec l'une ou l'autre : caigereh/queigereah (mélange). Chez Cillart ${ }^{62}$ (français-breton), l'entrée K est minimaliste, l'auteur précisant que $\mathrm{CA}$ est préférable à $\mathrm{KA}$ et que c'est « un reste de vénération pour l'Antiquité » qui conduit à écrire K barré les noms en Quer-. Le parti-pris de réalisme phonétique de Cillart (explicité dans son introduction et son projet de réforme de l'orthographe française) conduit à penser que dans un exemple comme : ne ouéss qué leignétt ? (vous n'avez pas dîné ? p. 254) le <qué> n'était pas palatalisé et que dans le cas contraire, il se serait étendu sur la question.

j) De 1768 à 1838, rien non plus (ou presque, cf. infra) dans les ouvrages des abbés Pourchasse et Marion ${ }^{63}$. Les vélaires sont toujours notées par les même graphèmes que précédemment et, sur ce point, les textes vannetais ou léonards ne se distinguent pas.

k) Plus aucun doute possible avec l'émergence de la linguistique moderne et de ses notations rigoureuses : en 1876, dans la description

60. Joseph Lотн, «Le plus ancien texte suivi en breton de Vannes », Annales de Bretagne, $\mathrm{n}^{\circ}$ 20-3, 1904.

61. Op. cit.

62. Op. cit.

63. Louis POURCHASSE, Instructioneu santell, Vannes, Galles, 1768 ; Buhé Sant Isidor, Vannes, Galles, 1787 ; Imitation hun Salver Jésus-Christ, Vannes, Galles, 1788 ; Jean MARION, Magasin spirituel er beurerion, Vannes, Galles, 1790 ; Instructioneu santel, Vannes, Galles, 1792 ; En Or ag er Vuhé devot, Vannes, Galles, 1838. 
du breton du bourg de Batz, par Léon Bureau, en dehors de quelques occurrences spécifiques (cf. infra), /k/, /g/, /sk/ et /sp/ ne sont pas palatalisés ${ }^{64}$.

1) En 1876 également, les palatalisations sont davantage présentes dans le breton de Sarzeau décrit par Ernault ${ }^{65}$. Mais des mots comme la particule négative ket, ou le verbe gwelet, voir, que l'on n'a guère de chance d'entendre aujourd'hui autrement que palatalisés, ne le sont pas encore à cette époque.

\section{Palatalisations attestées}

Un deuxième ensemble de documents laisse pourtant entrevoir que la palatalisation est bien une réalité depuis assez longtemps. Mais l'opposition entre vélaires et palatales semble très subtile et donc décrite avec embarras et de façon quasiment cryptée. Ce qui explique sans doute qu'elles aient peu soulevé l'attention.

m) En 1732, Grégoire de Rostrenen, indique dans son dictionnaire ${ }^{66}$ :

« La lettre $\mathrm{Q}$ a le même son chez les Bretons, \& se prononce aussi fortement que le k hormis dans Vannes \& dans la HauteCornoüaille où on prononce le q comme les François. Delà vient qu'un Breton prononce ces mots François : quel, quelle, qui, quinte, quinze \&c, comme si l'on écrivoit, kel, kelle, ki, kinte, kinze \&c. »

Grégoire, comme son nom l'indique, vient de Rostrenen, sur les marges entre Cornouaille et Vannetais. Il a été ordonné prêtre à Saint-Malo et a séjourné à Pornic. Il a donc eu l'habitude d'entendre le français de l'ouest et d'en mesurer la proximité et la distance avec

64. Lucien Bureau, « Le dialecte du Bourg de Batz », Revue celtique n 3, 1876. $<$ keid $>$, $<$ keit $>$ et $<$ ke $>$, pas (lignes 19, 21, 28, 30), <nikẽn $>$, personne (1. 16), $<$ tamik $>$, peu (1. 17). On relèvera aussi une évolution $/ \mathrm{a} />/ \mathrm{a} /$ qui bloque la palatalisation dans $<$ kamere $>$, prenez (1. 19) ; <ger $>$ et $<$ ger $>$, maison (1. 28, $30)$; < kegen-ẽ>, avec moi (1. 31) ; <paskə>, parce que (1. 32) ; < zisplas $>$ et $<$ displaseit $>$, se dépacer $(1.15,18,20)$, < respõdeit $>$, répondu (1. 29). Il y a aussi plusieurs occurrences de /g/ renforcés en $/ \mathrm{k} /$ par sandhi : $<\mathrm{kə}>,<\mathrm{ke}>,<\mathrm{ked}>$, avec (1. 29 et 30).

65. Émile ERnaUlt, « Le dialecte vannetais de Sarzeau », Revue celtique ${ }^{\circ} 3,1876$.

66. Tanguy GRÉGOIRE DE Rostrenen, Dictionnaire françois-celtique ou françoisbreton, Rennes, Vatar, 1732, préface. 
le breton qui lui était familier. Quelques années plus tard, dans sa grammaire, il réitère la même information, s'agissant cette fois des graphèmes $<$ gue $>$ et $<$ gui $>$ :

«C'est le g gamma des Grecs, \& le gaïn des Arabes. Ainsi que l'on prononce plus ferme que les mots françois, guérir, guerre, guide, guitarre $\& c^{67}$. »

Ces remarques peuvent dérouter un francophone d'aujourd'hui, qui a sans doute le sentiment de prononcer de manière identique le $<\mathrm{k}>$ et le $<\mathrm{q}>$, soit [k]. Or, pour Grégoire, il existe dans le français de son temps une prononciation forte (ou ferme), représentée par $<\mathrm{k}>$ et une prononciation faible (bien qu'il ne la nomme pas), représentée par $\langle\mathrm{q}>$. Le breton du nord-ouest ne connaît que la forte et la généralise, tandis que le vannetais partage le système binaire $\mathrm{du}$ français. Que faut-il comprendre par-là ? Vraisemblablement que l'articulation faible (avec $<\mathrm{q}>$ devant $<\mathrm{e}>,<\mathrm{i}>,<\mathrm{in}>$ ) représente une variante palatalisée, tandis que la forte $<\mathrm{k}>$ serait purement vélaire. Soit une subtile opposition de timbres : [k']-[k].

n) Cette supposition semble confirmée par l'introduction du Vocabulaire nouveau de l'abbé Marion ${ }^{68}$ (édition posthume). S'agissant des lettres $k$ et $q$, l'auteur explique que «l'usage commande que ces lettres se prononcent quelquefois serrées et comme entre les dents, (ce qui arrive toujours quand elles sont immédiatement précédées d'un $i)^{69} \gg$. Manifestement, on se trouve là devant une tentative de description d'une prononciation dorso-palatale, qui est systématique dans la finale /ik/.

o) Si en 1836, la grammaire de Guillôme déjà mentionnée en e) ne donnait pas de palatalisation pour $/ \mathrm{k} / \mathrm{ou} / \mathrm{g} /$, l'auteur précisait que le $<\mathrm{s}>$ « au milieu des mots se prononce comme ch : er voestr, les maîtres $»{ }^{70}$. Il s'agit là de la première description d'une palatalisation systématique du groupe $/ \mathrm{st} /$.

67. Tanguy GRÉGOIRE DE RoSTRENEN, Grammaire françoise-celtique ou françoisebretonne, Rennes, Vatar, 1738, p. 6.

68. Jean MARION, Vocabulaire nouveau, Vannes, Galles, 1829.

69. Le texte breton correspondant traduit presque littéralement le français et n'est donc pas beaucoup plus clair : èl ma oulen en usage ma vou prononcet maraùéh el lettrenneu-zé sterd hac èl étré en dènt (er péh e arrihue perpet a pe vai un i just én ou raug).

70. Op. cit., p. 7. 
p) Il faut toutefois attendre 1878, pour que la description gagne en intelligibilité : la grammaire de l'abbé Le Bayon ${ }^{71}$ indique alors :

«Il convient d'observer qu'il y a deux prononciations du K et du $\mathrm{G}$ devant $\mathrm{E}$. Ces lettres peuvent être dures, comme, en français, dans les mots quenouille, guenille, — ou mouillées, c'est-àdire, prononcées comme si elles étaient suivies d'un i, comme, en français, quérir, guérir. Les uns prononcent kér, ville; les autres, kier, er giér. »

Intelligibilité toute relative cependant : ici encore, l'explication repose sur un parallélisme avec une opposition en français, aujourd'hui inopérante (du moins en français standard).

Les graphies phonétiques permettent de remonter un peu audelà, mais de façon désordonnée, en fonction des phénomènes. Le groupe /st/ est le premier à pouvoir être observé :

q) Dans le formulaire de prône vannetais de $1631^{72}$, le mot chrétiens (mod. kristenien), apparaît sous la forme chrichenion, avec donc évolution de /st/ (avec [t'] palatal) en [S].

r) En 1723, Châlons ${ }^{73}$ reprend ce terme, en localisant ce type de palatalisation à Arradon et à Sarzeau, tandis que les formes en usage dans l'intérieur des terres sont graphiées $\langle$ st $>$. Ceci prouve seulement que le /t/ y était encore prononcé, mais faut-il lire les $<\mathrm{st}>$ de 1723 comme [st] ou [st] ? Le témoignage de Guillôme en 1836 (cf. supra) indique une généralisation de [ $\mathrm{ft}]$ un siècle plus tard, sans remise en cause de la graphie étymologique.

s) La généralisation de [jt] est confirmée en 1876 par Léon Bureau à Batz ${ }^{74}$ et Émile Ernault à Sarzeau ${ }^{75}$ et en 1886 par Joseph Loth pour tout le vannetais ${ }^{76}$.

71. A.-M. LE BAyOn, Grammaire bretonne du dialecte de Vannes, Vannes, Lafolye, 1878, p. 2.

72. Op. cit.

73. Op. cit.

74. Art. cit. Avec les exemples suivants : <daštümeit>, amassé, 1.13 ; <doštat>, approcher, $1.25 ;<$ mištẽ $>$ meilleur, 1.27 et $30 ;<$ ašti $>$, voici, 1. 24, 25, 29 et 32.

75. Art. cit. <chtágët>, attaché, p. 48, 1. 10 ; <fiecht>, fête, p. 49, 1. 5.

76. Joseph Lотн, "Remarques sur le bas-vannetais », Revue Celtique n 7, 1886. Page 178, l'auteur déclare : «L's [en bas-vannetais] comme en haut-vannetais a le son du français ch (cheval) devant $t$. » 
Les attestations de la palatalisation de $/ \mathrm{k} /$ commencent plus tardivement, mais elles sont ensuite beaucoup plus explicites. Les premiers craquements dans l'uniformité de la graphie affectent un cas particulier du groupe $/ \mathrm{sk} /: / \mathrm{s} /+/ \mathrm{k} /$ à la jonction entre deux mots, en l'occurrence le verbe avoir à la forme négative (mod. n'en deus ket, il n'a pas) ${ }^{77}$.

t) Si l'abbé Marion est fidèle aux graphèmes étymologiques, dans le Magasin spirituel er beurerion, face à 176 quet $^{78}$ il laisse échapper 3 chet $^{79}$. C'est évidemment bien peu (1,6\% du total), mais il est difficile de croire que l'on tienne là un reflet de la pratique. Il est bien plus vraisemblable qu'il s'agisse d'actes manqués, trahissant l'écart grandissant entre écrit archaïsant et oral qui palatalise déjà assez couramment.

u) En 1817, le manuscrit de la Vie de sainte Julitte (cité par Raude ${ }^{80}$ ) présente aussi de telles graphies évoluées et montre d'autres alternances, comme $<$ chueh $>$ et $<$ scueh $>$, fatigué.

v) En 1818, l'emploi de chet avec avoir, au lieu de quet, est systématique chez l'abbé Gicquello dans son Histoer a vuhé JésusChrouist $^{81}$. Le phénomène est d'autant plus remarquable que l'auteur se signale par les libertés qu'il prend : son orthographe s'écarte nettement du vannetais normé de l'époque et reflète la prononciation de la région de Lorient, où le livre est d'ailleurs imprimé, hors des circuits habituels contrôlés par l'Église.

w) La production littéraire vannetaise due aux ecclésiastiques utilisant le standard épiscopal reste fidèle plus longtemps au gra-

77. En revanche, on n'observe pas le même phénomène avec les autres verbes à d'autres temps : ne chomas quet, il ne resta pas (in Joseph LE THUAUT, Er fetcenieu a vuhé, Vannes, Galles, 1877, p. 29) ne montre pas de simplification en [S], ni à l'époque, ni dans les parlers d'aujourd'hui.

78. Op. cit., Ne mès quet a boén, je n'ai pas de peine (p. 2); ne hoès quet ivet, vous n'avez pas bu (p. 33), etc.

79. Op. cit., Ne mès chet er batiantced ém poénieu, je n'ai pas de persévérance dans la douleur (p. 71) ; Ne mès chet neoah caz doh Doué, je n'ai pourtant pas de haine envers Dieu (p. 81) ; N'en dès chet paud amen, il n'y en n'a pas beaucoup ici (p. 204).

80. A. RAUDE, art. cit.

81. J. GiCQuello, Histoer a vuhé Jésus-Chrouist, Lorient, Le Coat-Sant Haouen, 1818 . 
phème étymologique : $1810: n^{\prime}$ en dès quet ${ }^{82}$, il n'a pas ; $1829:$ n'ou dès quet éellet ${ }^{83}$, ils n'ont pas pu ; 1839 : ne hès quet ${ }^{84}$, il n'y a pas ; 1843 : n'hun nès quet ${ }^{85}$, nous n'avons pas. Mais vers le milieu du XIX ${ }^{\mathrm{e}}$ siècle, le remplacement de quet par chet est acté : 1845 :

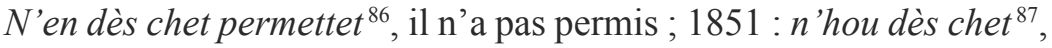
qui n'ont pas ; $1856:$ n'en dous chet ${ }^{88}$, tu n'es pas. Les textes ultérieurs consacreront le nouvel usage. On observe le même type de transition avec un mot comme diskenn, descendre, écrit $<$ disquen$n e t>$ (descendu) en $1819^{89}$ et $<$ dichennet $>$ dès $1829^{90}$. Les graphies sont pourtant hésitantes : en 1839, on lit encore < disquennet $>$ dans la Buhé er Scent ${ }^{91}$, mais surtout, on voit apparaitre un temps une sorte de graphème transitoire $<\mathrm{sch}>$, peut-être pour rendre compte d'un son affriqué [sc] encore instable : $1817^{92}$ : me meschett eunn, je n'ai pas peur ; ne chongeschett, tu ne penses pas ; 1818 : e zischenas ${ }^{93}$, descendit ; 1845 : e zischen ${ }^{94}$, qui descend. Ces hésitations se remarquent également avec la finale /ik/ : au lieu des classiques graphèmes $<-\mathrm{iq}>$ ou $<-\mathrm{ic}>$, on lit pinhuid, riche et yaouantis, jeunesse, chez Marion $^{95}$, ou quich, chair, chez Gicquello ${ }^{96}$.

x) En 1876, à Batz, L. Bureau ${ }^{97}$ indique de même que le /k/ n'est

82. Collectif, Catéchisme breton de Vannes, Vannes, Galles, 1810, p. 8.

83. Joachim GulLlôme, Imitation er Uirhiés glorius Vari, Vannes, Galles, 1829, p. 75.

84. QuiLlÉRÉ, Buhé er Scent, Vannes, Galles, 1839, p. 15.

85. Le Diot, Livre Brédiah er fé, Vannes, Galles, 1843, p. 15.

86. BELleC, Guirionéeu ag er religion, Vannes, Galles, nouvelle édition, 1856, p. 134.

87. DrÉANO, Catéchim historik en abad Fleury, Vannes, de Lamarzelle, 1851, p. 4.

88. LabOURLETTE, Guerzenneu eid oll er blai, Vannes, Galles, 1856, p. 38.

89. Anonyme, Er règl ag en drivet-Urh a benigen, Vannes, Galles, nouvelle édition, 1831, p. 12.

90. J. GuilLÔME, op. cit. (1829), p. 70.

91. QUILLÉRÉ, op. cit., préface, p. V.

92. Vie de Sainte Julitte (cité par RAUDE, art. cit.).

93. Op. cit. p. 6.

94. LABOURLeTte, op. cit. (p. 8-9).

95. Pinhuid: MARION, Officeu eid er sulieu ha gouilieu principal ag er blai, Vannes, Galles, 1849 (posthume, rédigé vers 1820), p. 27 ; Yaouantis : MARION, Instructioneu santél, op. cit., p. 107.

96. GICQUELlo, op. cit., p. 15.

97. Art. cit. 
palatalisé que dans ce seul contexte, avec évolution en [C]: $<$ ha hu ez še>, et vous n'avez pas (1. 29).

y) Toujours en 1876, les palatalisations, légèrement plus développées à Sarzeau ${ }^{98}$, concernent aussi le verbe avoir : $<$ ne os chet>, vous n'avez pas (p. 49, 1. 17). Mais cette fois, le groupe /sk/ évolue également en [ $\mathrm{jk}]$ dans <'́guchket $>$, habillez (p. 49, 1. 3) ${ }^{99}$. Cet exemple $<$ guchket $>$ montre que le $/ \mathrm{g} /$ peut être palatalisé seul. C'est aussi le cas dans $<$ beḱkolienn $>$, grande (p. 48, 1. 8), dans les finales en /-ik/: <iin tamek'>, un peu (p. 48, 1. 22) et dans l'emprunt au français dialectal < vëkêt $>$, biquette (p. 49, 1. 18).

\section{Une évolution articulatoire très récente}

Les sources citées jusqu'à présent montrent donc l'émergence et l'extension progressive d'articulations palatales entre le début du XVII et la fin du XIX ${ }^{\mathrm{e}}$ siècle seulement. Les sources ultérieures ne font que confirmer la dynamique. Mais elles rendent compte également d'une importante mutation : le passage d'une articulation dorso-palatale à apico-palatale.

Bien que maladroitement exprimée, la prononciation dorsopalatale [c] pour / $\mathrm{k} /$ étymologique se déduit aisément depuis Grégoire en 1732 jusqu'à Le Bayon en 1878. En 1886, Loth la décrit plus précisément comme « un $k$ iotacisé » ${ }^{100}$, tout en en donnant un exemple ambigu : « analogue par la prononciation au français $c$ dans cour ", nuance qui ne s'entend plus dans le français non-dialectal d'aujourd'hui. En 1914, Thibault reprend le même exemple pour Cléguérec ${ }^{101}$. Mais cette fois, il n'y a plus de doute, quand il précise que « $\langle\bigotimes\rangle$ arrive à ne plus guère se distinguer de $\langle\bigotimes\rangle$, surtout devant $e »^{102}$. Une telle prononciation iotacisée n'est d'ailleurs pas une spécificité vannetaise, puisque Loth la note également à Beuzec-Cap Sizun et Plogoff en 1898.

98. Art. cit.

99. En revanche, il conserve l'articulation [sk] dans < biskoac'h>, jamais, p. 49, 1. 17. 
Mais le vannetais a entamé une évolution qui consiste à pousser l'affriction de $/ \mathrm{k} /$ à son terme logique, c'est-à-dire [t]]. Et cette évolution commence par la façade maritime. Dès 1876, à Sarzeau, Ernault ${ }^{103}$ indiquait que les symboles des palatales de son système de transcription devaient se lire comme «tch et $d j »$. À Quiberon, en 1895, Loth explique que l'on entend « des palatales extrêmement iotacisées, qu'on pourrait prendre pour $t c h$ et $d j »$, ajoutant en note : «de fait, on peut s'y tromper et on s'y est trompé » ${ }^{104}$. Quinze ans plus tard, Le Roux, enquêtant pour l'ALBB, note encore essentiellement des palatales [c] et [f] dans tout le vannetais, sauf justement à proximité de Quiberon, qui est l'un des rares points où figurent des affriquées explicites [t] et [d $]^{105}$. Encore une quinzaine d'années plus tard, en 1927, J. Buléon écrit cette affirmation surprenante : « un signe qui fait reconnaître (...) un indigène de la Bretagne bretonnante $(. .$.$) : qui, gui se prononcent t c h i, d j i »{ }^{106}$. À partir de ce moment, toutes les descriptions du breton vannetais rendront compte de la palatalisation de façon analogue : la décrivant comme une affriction apico-palatale. En 1951, Falc'hun estime que « au pays de Vannes, rien n'est plus courant que d'entendre prononcer [ృчin ЈЧعn] ${ }^{107} \mathrm{ou}$

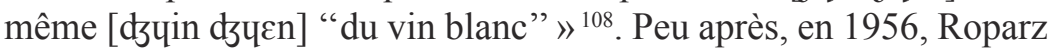
Hemon n'utilise plus que les signes $<\mathfrak{t}>$ et $<d_{3}>^{109}$ (1956: xxxii). En 1976, L. ar Bras transcrit le breton du Croisty en alphabet courant, utilisant pour les palatales les graphèmes $<\mathrm{tch}>$ et $<\mathrm{dj}>{ }^{110}$. Dans les années 1990-2000, je n'ai entendu à Malguénac que des [t] et [d]

103. Art. cit.

104. Joseph Loth, « Le breton de Quiberon », Revue celtique $\mathrm{n}^{\circ} 16,1895$.

105.ALBB, c. 102, tuchantik, bientôt ; c. 386, kig, viande ; c. 388, kegi, coqs. Les autres points concernés sont à peu de distance de là : c. 358, begin, deuil et c. 410, ozeganed, lutins : Ploemel ; c. 367, kegel, quenouille : Quiberon et Belle-Île-enmer; c. 459, mirvig, mie : Quiberon, Île-aux-moines et Locmaria (en Belle-Île); c. 527, pinvidik, riche : Quiberon, Ploemel, Île-aux-moines et Houat.

106. Cité par RAUDE, art. cit.

107. Je transcris en API, faute de disposer du signe correspondant à [d] en alphabet Rousselot-Gilliéron.

108. Op. cit., (Histoire de la langue bretonne...), p. 195. L'ouvrage fut publié en 1963, mais il reprend la thèse de l'auteur, soutenue en 1951.

109. Roparz Hemon, Christmas Hymns in the Vannes Dialect of Breton, Dublin, Dublin Intitute for Advanced Studies, 1956, p. xxxii.

110. L. AR Bras, « Mari, amzer eurus meche paset me bourap », Hor Yezh n ${ }^{\circ} 110$, 1976. 
et c'est cette valeur que j'ai donnée dans ma thèse aux signes /c/ et /y/. Je n'ai personnellement relevé les sons [c] et [f] qu'à Arzano ${ }^{111}$. Enfin en 2007, Cheveau indique que dans la région lorientaise, les locuteurs les plus âgés prononcent $[\mathrm{c}]$ et $[\mathrm{f}]$ et les plus jeunes [ $[\mathrm{g}]$ et $[\mathrm{d} 3]^{112}$.

\section{Conclusion}

La palatalisation vannetaise pose donc un défi au linguiste : elle semble être une tendance phonétique ancienne dans le sud-est de la Basse-Bretagne, mais une tendance dynamique, qui a connu des évolutions constantes et parfois très récentes. Au vu de tous les éléments apportés dans ce travail, il est possible de retracer son évolution comme suit :

La palatalisation résulte d'une lente migration de l'articulation des vélaires vers l'avant de la bouche, sous l'attraction des voyelles antérieures et médianes. En termes articulatoires, les vélaires furent d'abord nécessairement des dorso-palatales (la partie arrière de la langue formant l'occlusion). Quant au lieu d'articulation, il fut dans un premier temps post-palatal, transformant $/ \mathrm{k} /$ en [k'], à peine distinct du [k] vélaire (idem pour la voisée /g/). De cette façon, il est possible que le phénomène resta longtemps très discret, au point de n'être pas toujours perçu par la plupart des locuteurs. Graduellement, cette articulation migra encore pour devenir médio-, puis pré-palatale, prenant le timbre [c]. À partir de ce stade, la palatalisation devient mieux perceptible, ce qui conduit Grégoire de Rostrenen à la noter en 1732 et Marion vers 1820. Il est donc frappant de constater qu'elle se développe en breton parallèlement à la même évolution en français de l'ouest, selon une chronologie qui, bien qu'incertaine, paraît relativement proche ${ }^{113}$. Parallélisme entre roman et brittonique, qui

111. Erwan LE PIPEC, « Les schémas accentuels en breton du sud-est », mémoire de DEA, Université Rennes 2, 2004, annexes ; E. LE PIPEC, «Étude pluridimensionnelle d'un parler : description, émergence et aspects sociolinguistiques du parler du breton de Malguénac », thèse, Université Rennes 2, 2008, p. 145.

112. Loïc CheveaU, «Approche phonologique, morphologique et syntaxique du breton du Grand Lorient (bas-vannetais) », thèse, Université Rennes 2, 2007, p. 79 .

113. K. RinGENSON, op. cit., p. 108. 
pourrait s'expliquer par l'action sous-terraine d'un substrat commun. En l'occurrence, gaulois : celui-ci aurait légué aux deux langues une articulation mouillée, terme qui ne doit rien à la salive, mais dérive de mollire : rendre souple. La palatalisation serait ainsi le résultat d'une articulation peu tendue commune à tout le nord-ouest de la Gaule. Celle-ci est d'ailleurs attestée par l'instabilité des voyelles, qui présentent en français dialectal, comme en breton vannetais, une forte tendance à la diphtongaison ${ }^{114}$.

Par conséquent, si la palatalisation de /k - g/ n'est attestée en breton que depuis le début du XVIII ${ }^{\mathrm{e}}$ siècle, si elle n'est détectable à l'examen des voyelles qu'à partir du Moyen Âge, il est fort possible que le phénomène était déjà amorcé plusieurs siècles auparavant. Mais seulement amorcé et passant encore largement inaperçu ${ }^{115}$. Les témoignages de plus en plus précis du phénomène au long du XIX siècle laissent penser qu'elle s'est renforcée de manière constante sur cette période, gagnant en audibilité à la fois par l'étendue des distributions concernées et par la netteté des timbres palataux. Le stade ultime de cette évolution fut le passage à une articulation apico-palatale [tg]-[d]]. Mais pour spectaculaire que soit une telle innovation, il faut souligner qu'elle est très récente : après avoir débuté sur la côte, elle ne se généralise qu'après la première guerre mondiale, sur un territoire semblant assez bien correspondre à l'ancien pays de Vannes.

La palatalisation de $/ \mathrm{k} /$ devant voyelle antérieure entraîna mécaniquement celle du groupe /sk/ en contexte semblable, donnant [sc], rapidement simplifié en [J]. C'est sans doute ce traitement de /s/ obstrué qui, par contagion, entraîna l'évolution /st/ > [Jt], vraisemblablement dans la deuxième moitié du XVIII siècle, puisque le phénomène était généralisé, et devant toute voyelle, dès 1836. Contagion

114. L'articulation tendue distingue les variétés hautes de français depuis le XVIe siècle, ce qui a entraîné la disparition des diphtongues. Mais l'articulation nontendue domine encore dans les patois ou dans les niveaux relâchés du français du Québec. Une prononciation peu tendue provoque une instabilité générale, d'où le caractère phonétiquement hyper-évolué du français par rapport au latin, mais aussi du breton vannetais par rapport au breton central.

115. Personnellement, avant la préparation de cet article, je n'avais jamais remarqué qu'en français, Josiane Balasko ou les journalistes de France 2 palatalisaient autant... 
qui s'étendit également à /sp/ > [ $\mathrm{p}]$, possiblement à la même époque. L'évolution du groupe /sk/ devant voyelle postérieure diverge de celle devant voyelle antérieure ou médiane, puisqu'elle donne [jk]. Mais celle-ci ne concerne qu'une zone à l'est d'un axe Noyal-Pontivy/Auray. Elle pourrait donc être plus tardive, ce qui conduit, outre sa ressemblance avec [ $\mathrm{ft}$ ], à l'attribuer comme pour /sp/ à la contagion de $/ \mathrm{st} />\left[\int \mathrm{t}\right]$. À noter que devant une pause ou une frontière morphologique, /sk/ voit souvent généralisée la même évolution que devant voyelle postérieure : soit $[\mathrm{sk}]$ ou $[\mathrm{k}]$ selon les régions, d'où [ $\left.\nabla k \mathrm{ku}^{\prime} \mathrm{sk \varepsilon t}\right]$ ou [ $\left.\nabla \mathrm{ku} \mathrm{j}^{\prime} \mathrm{k} \varepsilon \mathrm{t}\right]$ (dormir).

Il est toutefois une autre source d'instabilité qui défie l'analyse. Raude ${ }^{116}$ et surtout Jackson ${ }^{117}$ témoignent ainsi de leur perplexité devant l'inconstance de la palatalisation : tel mot peut être palatalisé dans telle région, mais pas tel autre, malgré des caractéristiques identiques; pour un autre mot, les régions qui palatalisent peuvent être différentes, voire en miroir, comme le montrent les cartes cidessous, d'après l'ALRP :
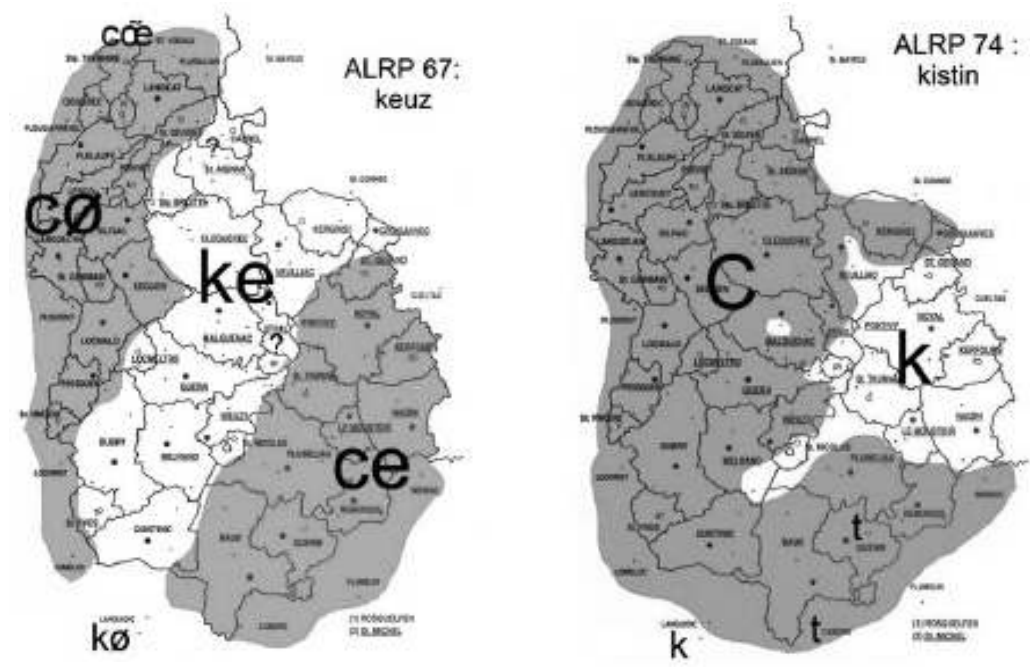

116. Art. cit.

117. Op. cit., p. 377 et 400. 
La palatalisation des $/ \mathrm{k} /$ initiaux est ici répartie quasiment à fronts renversés et aucun argument linguistique (phonétisme, étymologie...) ne paraît pouvoir l'expliquer. Au niveau individuel aussi, la palatalisation semble encore à ce jour d'ordre tendanciel et n'est pas stabilisée : pour certains mots elle est générale, pour d'autres, un même locuteur peut employer concurremment vélaire et palatale (surtout si une variation stylistique s'ajoute à l'usage local). Or, dans un certain nombre de cas, la solution pourrait être à rechercher dans la rétractation de l'usage et l'ethnicisation des variantes. La palatalisation a connu son développement maximal dans le temps même où le français se généralisait dans la société bretonnante. Au fur et à mesure du processus, le breton était de plus en plus cantonné à un usage local et familier. Voyant réduite sa fonction de communication au profit de celle d'identification, il devenait donc le lieu d'expression privilégié du narcissisme des petites différences: l'usage d'une forme pouvant devenir emblématique d'un terroir. Ainsi, les locuteurs peuvent palataliser tel mot mais pas tel autre, simplement parce que leurs voisins palatalisent à l'inverse d'eux et que c'est cette différence qui leur permet de s'en distinguer. L'anarchie apparente de certaines cartes pourrait donc s'expliquer non pas toujours par une logique linguistique, mais parfois purement ethnographique. 